\title{
CONFLICTOS TERRITORIALES EN LA COMUNIDAD DE WASAKÍN, MUNICIPIO DE ROSITA, REGIÓN AUTÓNOMA ATLÁNTICO NORTE (RAAN)
}

\author{
Autor: Pío Alejandro García Izaguirre ${ }^{[1]}$
}

\section{Resumen}

Este estudio de caso sobre conflictos territoriales en la comunidad de Wasakín, municipio de Rosita Región Autónoma Atlántico Norte (RAAN), fue descriptivo, en el análisis y valoración a dichos problemas hasta el año 2006, se han descrito las consecuencias y propuestas para darle salida al conflicto. Es un estudio de caso de Antropología Cultural, con el auxilio de las ciencias de la Etnografía, Historia, y Geografía para estudiar conflictos ocasionados por el recurso tierra.

Los resultados revelan que el principal factor de los conflictos lo han constituido los mestizos procedentes del Pacífico y Centro de Nicaragua, quienes llegaron entre 1916, 1957, en busca de mejores condiciones de vida. A partir de entonces, se han generado otras olas migratorias (1990), que han provocado conflictos socio-ambientales por la competencia de los recursos, la tierra, bosques y ríos en contextos sociales asimétricas. En lo político, los conflictos han tenido sus raíces en el Estado Nacional por el irrespeto e incumplimiento a los derechos de los pueblos indígenas, al ceder la explotación de los recursos naturales por extranjeros, principalmente por la ausencia de delimitación y titulación formal de sus territorios.

Palabras clave: Conflictos territoriales, conflictos entre familias mestizas y mayangnas, comunidades indígenas, procesos, autores locales internos y externos.

\section{Summary}

This study of territorial conflicts in Wasakin community, municipality of Rosita, North Autonomous Atlantic Region (RAAN), was mainly descriptive; the analysis and assessment of such problems until 2006, have described the consequences and proposals to be able to find a solution to the conflict. It is a case study of Cultural Anthropology, with the assistance of the sc iences of Ethnography, History, and Geography to study conflicts that are caused by land resource.

The results reveal that the main factor of conflicts have been the Mestizos from the Pacific of Nicaragua, who arrived between 1916 and 1957, seeking for better living conditions. Since then, other waves of immigration have been created (1990), which

[1] Máster en Antropolologia Social. Coordinador URACCAN-IEPA Las Minas. palejandrogi@yahoo.es 
have caused social and environmental conflicts for the competition of resources, land, forests, and rivers in an asymmetric social context. Politically, the conflicts have been rooted in the national government due to the disrespect and violation of the rights of indigenous peoples, by handing over the exploitation of natural resources to foreigners, mainly by the absence of formal demarcation and titling of their territories.

Keywords: Territorial conflicts, conflicts between Mestizos and Mayangnas families, indigenous communities, processes, internal and external local authors.

\section{Introducción}

Este estudio de caso aborda el conflicto por el uso del territorio de la comunidad indígena mayangna tuahka $\mathrm{k}^{[2]}$ de Wasakín y las familias mestizas inmigrantes. Esta comunidad está ubicada a $13.5 \mathrm{~km}$. al sur de Rosita, en la Región Autónoma del Atlántico Norte de Nicaragua, y a 480 km. de Managua. Su área comunal es de 47,538.01 hectáreas, con una población de 1,007 habitantes tuahkas y miskitus.

El propósito principal de la investigación es identificar los factores históricos que han generado los conflictos interétnicos por la propiedad en la comunidad; así mismo valorar sus consecuencias y el rol de la dirigencia indígena en la búsqueda de solución a los problemas.

Esta investigación pretende dar respuestas a muchas preguntas generadoras, tales como: ¿Cuál es el principal atractivo de las tierras comunales para la población mestiza inmigrante? ¿Cuáles son los factores históricos y culturales que generan conflictos con la comunidad? ¿Cuáles son los principales valores ancestrales que los indígenas reconocen y se disponen defender? ¿Cómo se piensa defender los recursos? ¿Cuáles son las consecuencias ambientales y sociales de estos conflictos? y ¿Cuál es la propuesta indígena y mestiza para su solución?

Desde la perspectiva de la Antropología Cultural, se analiza un conflicto de actualidad, de gran importancia para el gobierno y las autoridades comunales, que pretende servir de herramienta e insumo mediante la comprensión del conflicto para la defensa de la propiedad comunal del pueblo indígena de la comunidad de Wasakín, de sus derechos humanos y, específicamente el derecho consuetudinario.

Se espera que los resultados de este estudio contribuyan a fortalecer la identidad, la interculturalidad y al rescate del territorio de la comunidad, aspectos que se enmarcan en la misión y visión de la Universidad de las Regiones Autónomas de la Costa Caribe Nicaragüense (URACCAN).

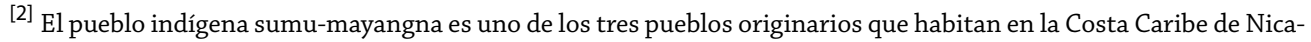
ragua, junto a miskitus y ramas. El pueblo sumu-mayangna tiene tres variantes lingüísticas: tuahka, panamahka y ulwas.
} 


\section{Revisión de la literatura}

En el análisis contemporáneo de los conflictos de propiedad hay dos perspectivas que se conocen a través de la historia. Una, desde la cultura occidental, basada en la propiedad privada; y dos, en la cultura indígena, basada en la propiedad comunitaria. En una segunda sección se conceptualiza el término conflicto, su significado, trascendencia y amplitud de acuerdo a las diversas perspectivas teóricas.

Los antecedentes de los conflictos por la propiedad de la tierra se identifican en todos los períodos históricos de la humanidad en el mundo. Para este proceso identificamos y referimos los siguientes:

Guerra (s/f), señala:

Hubieron momentos en la historia de la Humanidad que no existió disputa alrededor de la tierra., "hace al menos 10 millones de años los seres humanos organizados en grupos pequeños, los hombres se relacionaban por vínculos sanguíneos. La propiedad de la tierra era común, trabajan colectivamente y la distribución era equitativa".

Paredes (1999), describe que posterior a la Revolución Francesa se instituyó el Imperio Francés (1804-1815), en el que: [...] "se declararon abolidas la servidumbre, el derecho a la caza y a las justicias señoriales se aplica: la distribución de la propiedad. Se dan transformaciones sociales, donde progresa una economía de subsistencia a una economía de mercado". (p. 65).

Hodgson (1999), escribe:

en América, para los pueblos originarios constituye el continente Ab'ya Yala, "[...] La sociedad Indígena no estableció la propiedad privada, por lo que en su interior tampoco existieron clases sociales antagónicas, aunque sí diferenciación social con su propia dinámica de desarrollo colectivista y de convivencia con su medio ambiente (p.14).

N. de Fernández (1975) indica una segunda causa cuando dice: "los europeos obligaron a los indígenas con mecanismos de explotación". "La mita” para el trabajo de la minería abandonando la agricultura para trabajar en la minería forzada (p.199).

Kreimer (2005) y Paredes (1999), plantean la cuarta causa que es la esencia del neoliberalismo. "[...] la globalización y al fenómeno de "desterritorialización". (Ibíd.10o). Se liberaron del régimen señorial, pero se vieron perjudicados por el retroceso de los derechos colectivos y de tradiciones comunitarias (p.214). 
Hardin (1968), manifiesta el problema que la gente necesita alimentarse para la obtención de energía. La población sueña con lograr poseer la estabilidad social. "[...], el día que se vuelva realidad largamente soñada la meta de estabilidad social. En este punto, la lógica inherente a los recursos comunes inmisericordemente genera una tragedia. El mismo autor argumenta: "la libertad de los recursos comunes resalta la ruina para todos".

Maclean, Melba (s/f), manifiesta:

Que en virtud de este criterio explicativo, el derecho de propiedad que se recoge en la Convención de América sobre Derechos Humanos sobre Awas tingni y la concesión del Sol del Caribe (SOLKARSA), debe interpretarse de forma amplia para dar cabida también a las formas de propiedad indígena de carácter comunitario, y no meramente individual; que reviste elementos no sólo económicos, sino también profundamente culturales; y que tienen como fundamento último el derecho consuetudinario de los propios pueblos indígenas de acuerdo con la Corte $(\mathrm{s} / \mathrm{f})$.

Tarrow (1997), y Kranz (2005) afirman: "que los conflictos surgen en las sociedades por diferentes causas y factores". Son varios los que estudiaron el tema. Kranz refiere: "los conflictos se dan en las sociedades por las relaciones interpersonales entre grupos internacionales, interculturales, interespecies, por bienes y valores". (pp.3, 4).

Stavenhagen (2000), afirma que:

"los conflictos interétnicos en el mundo se dan por la lengua, la religión y el territorio". Enfatiza que "los conflictos se dan por relaciones asimétricas, cultura, tierra, economía, por política, laborales, étnico y religioso. En relación al problema de la tierra”. "los conflictos se dan cuando la relación entre grupos étnicos es asimétrica". (s/n).

Vélez (2000); para el analisis de conflictos plantea la siguiente teoría:

Los conflictos se dan interés por la distribución de recursos, para reconocimiento de poderes, el prestigio, la inducción, oportunidades de desarrollo, seguridad patrimonial. De validez cuando hay confrontaciones por creencias, dilemas, conducta, culturas, y de derecho se dan en torno a la aplicación de normas o regulaciones, modificaciones de marcos legales por ser acuerdos a nuevas realidades y que aplica enfoques diferentes. 
Kranz (2005) plantea: un análisis histórico de su desarrollo y confrontación de las diferentes formas propiedad, que son resultado de las diversas concepciones y cosmovisiones (pp.3-4).

Marroquín M. E. (1987), explica:

Indudablemente, otra de las causas de los conflictos territoriales han sido las reformas agrarias implementadas por los gobiernos en América Latina, que no han respondido a los derechos de los pueblos indígenas con respecto al derecho al recurso natural tierra (p. 15).

El Centro de Apoyo a Programas y Proyectos (CAPRI) (1998), en el estudio describe:

Con referencia a la incidencia de las políticas agrarias, el Centro de Apoyo a Programas y Proyectos documenta que en los años 70 el IAN impulsó el tercer proyecto de envergadura en la región -el proyecto Siuna-, de 35,0oo hectáreas, localizada en el triángulo de los ríos Silbí, Kipó y Prinzapolka. Se asentaron un promedio de 800 familias campesinas, adjudicándosele lotes de 50 manzanas a cada una.

Carlos, V. (1989) investigó:

el Instituto Agrario de Nicaragua (IAN), inició así grandes proyectos de "colonización" para beneficiar a familias individuales y otorgar títulos de tierras a los beneficiarios por ejemplo, que entre 1964 y 1973, el IAN tituló a 16 mil familias, otorgando un promedio de 65 hectáreas por familia, y que la gran mayoría de las tierras tituladas se ubicaban en la "frontera agrícola". (p.67).

Hale (1998), precisó: Consistente en lo anterior, ninguna de las dos leyes introdujo disposiciones especiales diferenciadas por las tierras indígenas, la ley de 1963 no hace referencia alguna al respecto y la ley de 1981 simplemente reafirma (arto 30) la "voluntad del Estado por disponer de tierras necesarias para las comunidades indígenas, para que la trabajen individualmente... Ambas legislaciones no reconocen, ni confrontan el problema, de que en muchos casos las comunidades indígenas reivindican como suyas las mismas tierras que el Estado considera tierras nacionales, a pesar que las comunidades carecen de documentos legales para respaldar sus demandas (pp.78-79).

Según Roldan (1996): "la concesión de un título comunal se justificaría en el mandato de asegurar que las tierras sean trabajadas "productivas y eficientemente" (artículo uno) de ambas leyes, sin ningún reconocimiento de derechos indígenas preexistentes" (p p. 31,79 y 80).

Gabriel (1993) refiere: "la reforma agraria en Nicaragua llevada a cabo por el gobierno sandinista afectó gran parte de las tierras de la comunidad indígena que 
se encontraban en manos de terratenientes" en el Norte y la Región del Este de la Costa Atlántica. En 1979 quedan confiscadas un millón seiscientas mil manzanas, dos millones ochocientos mil manzanas entregadas a 100 mil familias durante siete años. En 1986 la reforma agraria se consolida.

Según Williamson, W. (1996): Lo anterior ayuda a explicar lo que se puede apreciar como una tendencia acelerada de responder a las demandas indígenas por la tierra a través de títulos comunales. Entre 1986 y 1990 MIDINRA concedió 8 títulos de esa índole, todos a comunidades sumus-mayangnas. Habría que añadir los títulos concedidos a los tres asentamientos de Tasba Pri (14,00o hectáreas), que también fueron concedidas como tierras comunales (Central American and Caribean Research Council CACRC, 1998: 80).

\section{Materiales y método}

La investigación se realizó en la comunidad de Wasakín, que es una comunidad indígena, además se tomó en cuenta tres comunidades mestizas vecinas y que han estado involucradas en conflictos por tierra con los indígenas. Inició en el mes de noviembre 2004 y concluyó en diciembre 2006. Como una distinción fundamental del enfoque cualitativo fue necesaria la aplicación del método inductivo, sobre todo en el análisis de las situaciones sociales descubiertas en las diferentes etapas estudiadas.

\section{Resultados y discusión}

En torno al tema de conflictos territoriales en la zona de Las Minas y Prinzapolka se han generado alrededor de 14 casos de conflictos por tierras. En cuanto a los conflictos por territorio en Rosita, resalta el problema enfocado en los sectores Dos Bocas de Kukalaya, donde aproximadamente 20 familias inmigrantes están afectando 29,000 manzanas de tierras. Esta problemática persiste desde 1991-1992 cuando el gobierno de Violeta Barrios de Chamorro designó tierras a los retirados del Ejército de Nicaragua (EN) y del Ministerio de Gobernación (MINGOB). En la actualidad el problema se agudiza con la Ley de demarcación territorial de las tierras comunales indígenas.

Otro conflicto es el que se vive en la comunidad de Yakalwas. En este sector el problema es de invasores a la propiedad indígena comunal. Los indígenas alegan que unas 240 familias procedentes de Mulukukú, San Pedro del Norte y Paiwas. Desde el 2003 tienen posesión de 2,000 manzanas dedicadas a la agricultura y explotación de madera, lo cual mantiene a estos dos grupos en constante pugna. En esta misma zona también están posesionadas en 20,000 manzanas de tierras indígenas unas 240 familias mestizas y retirados del Ejército de Nicaragua.

Asimismo, en el sector de las comunidades de Sansanwas, Wasakín y Empalme de Alamikamba. En el lugar llamado Misalaya, comunidad de Sansanwas, a unos 40 


\section{CIENCIAS SOCIALES}

kilómetros de Rosita, existe otro conflicto de tierra que afecta 5,000 manzanas de tierra posesionadas por unas 20 familias (ex policías, ex ejército nacional y otros). Existen 2 escrituras de la tierra con diferentes dueños. Siempre en Rosita en el sector Samaria, El Rosario y el Buen Samaritano, todos de Kukalaya están afectadas 5,000 manzanas, donde están posesionadas 80 familias mestizas desde el 2003, organizados en colectivo, en su mayoría son originarios de río Blanco, Matiwás y Mulukukú.

Varios ciudadanos" negociantes de tierras" forman parte del conflicto como precaristas en contra del colectivo de origen miskito, que tienen posesión de las tierras bajo el argumento que tradicionalmente han sido comunales.

En el triángulo minero existe la discriminación racial, la inadecuada administración de justicia en las demandas principales por invasiones de tierras, por lo general en los juzgados no les ponen atención a los planteamientos de los indígenas, las autoridades siempre han considerado que los indígenas no poseen límites territoriales estables, ni mapas de sus comunidades, podría ser que los demandantes tengan o no razón de sus quejas, por esto es que hay que aplicar la ley No. 445 (2003).

Factores que influyen en los conflictos territoriales en Wasakín: Uno de los factores que han generado los conflictos en la comunidad de Wasakín ha sido narrado en varias ocasiones por personas honorables de la comunidad ${ }^{[3]} \mathrm{y}$ al respecto refirieron: "los conflictos territoriales en la nación tuahka aparecieron con la llegada de los mestizos en diferentes oleadas migratorias de carácter laboral, motivados por la apertura de Las Minas en el municipio de Rosita".

Una segunda oleada migratoria se inicia en la década de los ochenta con la guerra. Además, que hay un desplazamiento de la población hacia el territorio costeño por causa de enfrentamiento militar, como producto de los acuerdos entre en Frente Sandinista de Liberación Nacional (FSLN).

En Wasakín en esos años, se reubicó a sumus-mayangnas de Bonanza en la comunidad de Kalmata, territorio de Wasakín, mientras duraba la guerra. Al finalizar el conflicto se resistieron a desalojar 1700 hectáreas ocupadas a esta comunidad. "los sumus de Kalmata tienen la costumbre de venderles tierras a los mestizos" (Neddy y Bler, 2005). Los sumus de Bonanza radicados en Kalmata estaban gestionando el desmembramiento de la comunidad núcleo.

La tercera invasión se da a partir de 1990 con el ascenso de la Sra. Violeta Barrios de Chamorro, que comenzó a repartir tierras, a crear polos de desarrollo para todos

[3] Neddy I. 2004; Montiel C. 2004; Watson 2004; Johnny S. 2004; Simon T. 2004; ex síndicos y ex jueces, y por (Smith P. 2005; Fredrick J. 2005; Bler P. 2005 y Simons T. 2005), ambos ex síndicos y ex jueces, fundadores de la comunidad. 
aquellos vinculado a la guerra del anterior gobierno. Se ubicó a esta gente en tierras como si fueran del gobierno, pero que en realidad eran áreas pertenecientes a los indígenas desde épocas remotas.

La cuarta oleada, se inicia con el gobierno de Arnoldo Alemán (1999 - 2003) y Enrique Bolaños (2003 - 2007). Esta oleada es "política", debido a que sus candidatos habían ofrecido tierras, títulos y contribuido al traslado masivos de campesinos de Matagalpa, Boaco y Chontales hacia tierras comunales en el triángulo minero, en Kukalaya, a cambio de votos para el Partido Liberal Constitucionalista (PLC).

Otro factor es que personas que han emigrado a la Costa Caribe lo hacen después de haber vendido a altos precios sus fincas, luego en el territorio de Las Minas han comprado tierras a precios ridículos, lo que permite a estos inmigrantes posesionarse de grandes áreas de tierra, ya sea tierras comunales o con títulos extendidos por el Instituto Agrario Nicaragüense (IAN) o mediante escrituras llamadas títulos supletorios, extendidos por abogados de la zona, porque incluso han extendido títulos después de publicada la Ley No 445 (2003), donde se prohíbe la ejecución de este tipo de trámite. (Entrevista con Rivas, 2006).

URACCAN CISA (2006), informa: Otro motivo de inconformidad que se destaca entre los indígenas es la presencia de extranjeros en el interior del territorio, como es el caso de un extranjero árabe, quien posee cuatro mil 100 hectáreas del territorio según los líderes indígenas, en 1999 cortó 750 árboles de caoba y los vendió". Esta finca abarca la comunidad de Ibu con 9 casas, 49 personas entre tuahkas y miskitus.

Las autoridades indígenas además de manifestarse divididas por factores de conflictos, funcionan por separado de acuerdo a intereses particulares, al extremo que permiten a los mestizos instalarse en territorios indígenas y después no pueden desalojarlos, porque luego estos se sienten dueños de la tierra.

El líder comunitario Simon A. ( 2006) describe: "cada autoridad comunal hacen arreglos de permisos para lo cual muchas veces falsifican papeles, y sellos que autorizan a los mestizos para trabajar en la agricultura o avales para extraer madera a cambio de estos unos piden un cerdo, maíz, frijoles o arroz, otros cobran $C \$ 200.00$ córdobas, una bicicleta o cualquier "chochada", y lo poco que se percibe en dinero y artículos solo beneficia al grupo que hizo la negociación mientras que la población no percibe ningún beneficio". Circunstancias que los mestizos han aprovechado para asentarse en tierras en forma permanente y no cumplir con los acuerdos, ya que de 35 familias a quien se le alquila tierra, sólo 7 solventaron impuesto y 28 jefes de familia han rehusado a cumplir el compromiso. 


\section{CIENCIAS SOCIALES}

\section{Al entrevistar a Fenly J. en 2006 expresó:}

otro tipo de relaciones interétnicas muy particular se da con San Francisco y Wasakín donde hay buenas relaciones de intercambio y mutuo aprovechamiento en el uso de la tierra. En cambio, la población de la comunidad de Cerro El Tigre sector [su/ Panhil] se rehúsa a cualquier compromiso con los indígenas. Estos han acaparado 10 mil manzanas de tierra y la utilizan para la extracción de madera, ganadería y en último lugar la agricultura y continúan invadiendo, carrilando, vendiendo tierras entre 50, 70, 100, y 3,000 manzanas. Estas ventas las hacen a mestizos que llegan de Matagalpa, Chontales, Boaco, Waslala, Siuna, (Las Quebradas) y Mulukukú.

Los conflictos por el uso de la tierra no solamente se dan con los mestizos, sino también existen conflictos intraétnicos con indígenas miskitus radicados en este territorio, que también quieren desmembrar Kubalaya, Walpatara e Ibus, situación que no es apoyada por las autoridades comunales de Wasakín. La Plywood, filial transnacional (PRADA), ha contribuido en el conflicto de tierras desatándose una fiebre de explotación de maderas en la comunidad.

La inadecuada administración de justicia es otro obstáculo que enfrentan los indígenas y que genera su inconformidad. Habría que agregar el desinterés por parte de la alcaldía, la policía, consejales regionales para resolver los casos que presentan los indígenas. El conflicto legal de la tenencia de la tierra por los mestizos

Los conflictos territoriales en la comunidad tienen su base en el generalizado desorden jurídico de la propiedad, que en esta zona es mucho más complicado. Los mestizos que viven en territorio indígena poseen títulos que fueron extendidos por:

- Títulos emitidos por el Instituto Nacional Agrario (IAN) en 1968.

- Constancias emitidas por los jueces de mesta desde los tiempos de Somoza,

- Constancias emitidas por los síndicos de la comunidad de Wasakín.

- Constancias efectuadas por las mismas juntas directivas de las comunidades.

- Documentos extendidos por políticos, la Unión Nacional de Agricultores. y Ganaderos (UNAG) y las juntas directivas de los síndicos.

- Constancias emitidas por ex dirigentes de la Unión Nacional de Agricultores y Ganaderos (UNAG).

- Títulos del Instituto Nicaragüense de la Reforma Agraria (INRA), emitidos por el Dr. Jaime Wheelock Román en la década de los ochenta. También los Comités de Defensa Sandinista (CDS), extendieron documentos para justificar la ocupación de tierras en territorio indígena. 
- Constancias de Reforma Agraria de 1992 a favor de los desmovilizados de la guerra.

- Así como Constancias emitidas por el Ministerio Agropecuario y Forestal y la Dirección General de Reforma Agraria de 1998, firmado por el Lic. Carlos Guerrero Bojorge, director de titulación a favor de los retirados de Asociación de Militares Retirados (AMIR).

- Avales extendidos por el Instituto Nicaragüense de Reforma Agraria (INRA) regional en 1988, firmados por Rosuela Gustavo Rosales, delegado de la institución en la RAAN.

- Constancia del INRA en Puerto Cabezas en 1994, por el delegado regional Alberto Escobar Tompson.

- Constancias por la secretaria de demarcación territorial del Gobierno Regional Autónomo de la RAAN en el año 2002, por Uriel Vanegas, director de la secretaria del gobierno regional.

- Aval firmado por el coordinador de gobierno Gustavo García Becker, y avalada por los ex combatientes de Yapti Tasba Marsaka Asla Takanka (YATAMA) Organización de los Hijos de la Madre Tierra (Rene Becker y José Dublón).

- Avales hechos por ex alcaldes en los noventas. Avales extendidos por el gobierno regional autorizado por Saberio Pantin.

- Escrituras de compra y venta hechas por abogados de la zona. Títulos supletorios emitidos por abogados de Siuna, del 2003-2006.

- Avales que extendieron los jueces de Cañadas llamados Jueces de Mesta durante la Administracion Somoza.

- Estos títulos de propiedad, permisos, avales o documentos extendidos por diferentes instancias a familias mestizas mantienen en constante pugna a las dos culturas, ya que ambas reclaman un determinado territorio.

Esta lista fue construida a partir de la información proporcionada en entrevistas con Rivas G. C. (2006), presidente y promotor de la ley No 445; Poveda R. Juan (2006), presidente de la UNAG, Rosita; Rodríguez, F. (2006), secretario político; Kiapa Wilson, P. (2006), delegado de Gobierno Autónomo, YATAMA de Rosita.

Producto de las relaciones sociales desiguales que se han venido construyendo entre indígenas de la comunidad y los mestizos inmigrantes, que se han establecido desde hace muchos años en tierras comunales, se han provocado consecuencias que generalmente son negativas dentro de las cuales las más relevantes son: 


\section{CIENCIAS SOCIALES}

\section{Las pérdidas de vidas humanas e inseguridad ciudadana:}

La destrucción de los recursos naturales, el medio ambiente y pérdida de territorios comunales han sido las principales causas del surgimiento de conflictos armados dentro de los que se describen los principales: Hace 10 años se originó un conflicto armado entre mestizos e indígenas de Wasakín por territorio, donde perdió la vida el indígena Herly Bendlis. Rodolfo Bendlis quedó inválido y Alberto Dolores herido con arma blanca.

La violencia e inseguridad se afirma con las invasiones de los mestizos, el sector Panhil [su /cerro El Tigre] a Wasakín, les respondieron a sus reclamos con frases como: "los vamos a guindar, los vamos a palmar a estos sumitos" (Johnny A. 2006). Otra expresión dicha por un mestizo, refiriéndose al problema de tierras y al tipo de títulos que poseen, resalta el nivel de agresividad que se vive, cuando un indígena le dijo "yo tengo la ley en mis manos" y el mestizo respondió: "¡métete, que yo tengo la escopeta!".

Los indígenas rompieron la paz que existía, hay rompimiento de relaciones entre mestizos e indígenas, amenazas de muerte por ambas partes. Incremento de la pobreza y mayor vulnerabilidad de los mestizos de San Francisco que no poseen tierras ante la actitud negativa de los indígenas en cuanto al alquiler de sus tierras porque no pagan el canon de arrendamiento (Johnny A., 2006).

Existe la amenaza de una posible guerra entre mestizos e indígenas, dijo Smith A. (2005):

que los jóvenes sumus mayangnas están dispuestos a hacer la guerra a los mestizos invasores de San Francisco y del sector Cerro El Tigre, que se encuentran en tierras comunales sin autorización de las autoridades principales de Wasakín. Están armados con arcos, flechas, armas blancas y rifles calibre 22".

La presencia de otra cultura en el entorno de Wasakín está incidiendo en la aculturación de los indígenas por lo que poco a poco van perdiendo su cultura conservacionista y prueba de esta afirmación es el uso de motosierras por parte de muchos indígenas con la que cortan madera para la venta. Según los indígenas: "los mestizos quieren joder a nosotros", "Wasakín ha puesto mojones y el árabe Kamel Benlos ha arrancado una y otra vez, lanzándolos al río” (líder comunitario, Simons T. 2004).

La situación económica ha empeorado porque las comunidades indígenas se han dedicado al comercio de madera en detrimento de la producción agrícola para el consumo (Entrevista a Neddy: 2006). Esta nueva coyuntura en los últimos 26 años ha conducido a los comunitarios a abrir nuevos cominos para la sobrevivencia en el 
comercio de madera y venta de permisos agrarios, que en lugar de resolver el problema, han creado otro tipo más complejo de conflicto.

Durante las entrevistas se descubrió que hay malas relaciones interétnicas entre mestizos e indígenas, motivados por el racismo y la discriminación. Existe resentimiento de los indígenas con el gobierno, en todos los niveles por la exclusión social en que los han mantenido (Entrevista Neddy; 2006). El roce de las dos culturas ocasiona otro tipo de conflictos como el abigeato, ya que se les están perdiendo animales domésticos. Además hay una invasión del ganado de los mestizos, el cual lo traen de la región central en camionadas y cuando la policía nacional recibe demandas de los indígenas, estos les dicen: "péguenle un tiro al que pase por su tierra".

La invasión de las tierras ha tenido consecuencias negativas Sobre los límites territoriales de la comunidad, dado que los carriles han sido violados por los mestizos invasores, lo que causa lamento en los indígenas que aspiran recuperar sus tierras para heredar a sus hijos. (Entrevista con el líder, Arguello, J.2006). Es evidente que a los mestizos lo que les llama la atención, son los recursos naturales de las tierras y bosques vírgenes, por ello los mestizos hacen todo lo posible por establecerse en estas áreas.

La propuesta principal generada tanto por los indígenas como por los mestizos es La aplicación de la Ley No. 445 ley del Régimen de la propiedad comunal para darle salida al conflicto de las tierras. Según los indígenas esta ley ayudará a los indígenas en garantizar la propiedad comunal. Para ello proponen para la defensa de sus tierras:

- Exigirle al gobierno el cumplimiento de la Ley No. 28, 445 y hacer uso de la Ley No. 540 Mediación y Arbitraje.

- Defender la identidad indígena, los símbolos de la iglesia, el mapa de la comunidad, los mojones instalados en 1967 , los carriles y hacer gestiones ante el gobierno para el retiro de los mestizos del territorio de la comunidad, además de adquirir áreas complementarias, que comprende hasta el estadio del municipio de Rosita.

- Se proponen fortalecer la organización comunitaria, incidir con el gobierno para que paralelo a la titulación de los indígenas, le titulen las tierras a los mestizos. La realización de foros e involucrar a todos los sectores políticos, sociales, religiosos, del gobierno, y no gubernamentales para analizar el fenómeno, consensuar para la equidad en la toma de decisiones.

- Presionar a la Comisión Intersectorial de Demarcación y Titulación (CIDT) a la Comision Nacional de Demarcación y Titilación de Tierras Indigenas (CONADETI), para aplicar un plan sobre mediación y resolución de conflictos de tierras con los comunitarios. 


\section{CIENCIAS SOCIALES}

- Involucrarse en el proceso en búsqueda de fondos y acompañamiento a la comunidad en la etapa de la presentación de la solicitud, con el diagnóstico bien elaborado.

- Definición de un plan de delimitación y legalización de la tierra de Wasakin en coordinación con la CIDT, CONADETI, así como la elaboración de un plan de divulgación de la ley No 445, radial, presencial y gráfica.

\section{Los mestizos proponen para defender su posesión:}

- Reformar la ley No 445 y su aplicación.

- Hacer cumplir las leyes No. 28 y la Ley No 540 "Ley de Mediación y Arbitraje".

- Defensa de sus tierras a corto plazo mediante la organización inmediata y la gestión ante el gobierno

- La titulación de las fincas en comunidades mestizas y la titulación a las comunidades indígenas.

- Durante el proceso debe prevalecer la mediación y arbitraje multiétnico con el gobierno, organismos no gubernamentales, políticos, sociedad civil, religiosas, militares, para el consenso y equidad en la toma de decisiones.

- Involucrar en un foro a todos los sectores políticos, sociales, religiosos, de gobierno, no gubernamentales para analizar el fenómeno, que haya consenso y equidad en la toma de decisiones.

- Presionar a la CONADETI y la CIDT, para formulación y aprobación por el pleno de un procedimiento para la resolución de conflictos entre comunidades en materia de demarcación.

- Acompañamiento de los territorios en su proceso de elaboración diagnóstica para los que ya tiene financiamiento gestionado. En el caso de los nuevos territorios ayudar a la gestión de esos fondos. Involucrarse en el proceso.

- Definir en coordinación con la CONADETI los territorios a demarcar en la siguiente etapa.

- Organizar un plan de divulgación de la ley No 445, radial y presencial gráfica.

\section{Principales hallazgos en la investigación}

Durante el desarrollo de la investigación se encontraron hallazgos que no estaban contemplados en los objetivos del estudio, dentro de los cuales se destacan:

Los conflictos intraétnicos entre la comunidad de Wasakín con indígenas miskitus que viven a lo interno de esta comunidad. Estos conflictos tienen dos tendencias: una dirigida al fraccionamiento del territorio indígena promovido por los miskitus; 
la otra, encaminada a la transformación de las tierras comunales en tierras privadas, siempre bajo la dirección de sumos y miskitos.

Existen buenas relaciones interculturales entre los indígenas de Wasakín y los comunitarios mestizos de San Francisco con más tiempo de vivir en la comunidad, a pesar de que muchas veces no se cumplen los acuerdos de arrendamiento de tierras. Los indígenas manifestaron que no tienen problemas con estos campesinos autóctonos, porque ellos son personas que respetan a los indígenas. En cambio, las relaciones con los mestizos con poco tiempo de estar en la zona no son cordiales, dado que esta nueva generación tiene intenciones de apoderarse de las tierras y día a día están invadiendo más terrenos indígenas por encima de la Ley 445.

Existen dirigentes indígenas de la comunidad de Wasakín que han contribuido en el incremento de los conflictos, debido a que estos negocian con los mestizos en la extensión de avales y permisos para la extracción de madera y trabajos agrícolas. Los indígenas jóvenes de la comunidad están perdiendo su cultura, ya que existen alrededor de 10 motosierristas que se dedican a la extracción de madera preciosa para luego comercializarla. Ellos no consideran importante darle continuidad al derecho consuetudinario; en cambio, los ancianos son quienes mantienen la cultura conservacionista y están luchando por preservar la identidad cultural.

Las autoridades comunitarias están divididas, no existe unidad y consenso, sobre todo en la toma de decisiones para avalar la extracción de madera, autorizándola de forma individual. Con referencia a las autoridades territoriales y comunales los mismos ex síndicos se refieren a la situación actual sobre este tema de carácter sociopolítico, Fenly J. Emilio (2006), explica:" son los mismos síndicos cómplices con los conflictos territoriales". Las diferentes instancias, tales como el juez, consejo de ancianos, los jóvenes actúan de manera independiente. De igual forma las comunidades a lo interno en la comunidad central, son la célula del divisionismo territorial y de iniciativas de propiedad privada.

Asimismo, se encontró que los profesionales originarios de Wasakín no están aportando al desarrollo de su comunidad, y están adoptando modelos culturales mestizos, incluso no regresan a vivir a ella, sino que se quedan en el municipio. Las mujeres no cuentan con el aval de sus padres para salir de la comunidad para continuar sus estudios universitarios. 


\section{Conclusiones}

Los conflictos que se identifican en la comunidad en Wasakín se tipifican como socio ambientales, intraétnicos, interétnicos, inspirados en la codicia de los recursos naturales tierra, bosques y agua por ambas partes: mestizos autóctonos y sumu-mayangnas.

La reforma agraria no ha dado respuestas a la seguridad de las tierras indígenas. La Comisión Nacional de Demarcación y Titulación (CONADETI), en cuatro años y con 11 millones de córdobas invertidos, tampoco ha podido dar respuesta a la problemática por el uso de tierra.

Hace falta presupuesto para la implementación de la ley No. 445, que apoye además a las comunidades indígenas en sus gestiones ante las instancias del gobierno para la agilización del proceso.

La discriminación racial, la inadecuada administración de justicia en contra de los pueblos indígenas, la ausencia de la participación de la mujer, la juventud y la sociedad civil en la toma de decisiones en el proceso, limita la agilización y la obtención de resultados satisfactorios.

El gobierno y los mestizos tienen una percepción negativa sobre la cultura del indígena lo consideran "holgazán", "que no les gusta trabajar", opinan que "para nada tienen esas inmensas extensiones de tierras y no las trabajan".

En el ámbito jurídico, ha existido un desorden e irresponsabilidad de todos los gobiernos en el siglo XX, con relación a la legalización de la propiedad dirigida y encausada a favor de los mestizos, afectando indiscutiblemente a la propiedad comunal.

Respecto al enfoque de género, la participación de la mujer en este proceso es poco visible o invisible. En Wasakín, los síndicos, los jueces y los miembros de los consejos, todos son hombres, no participan mujeres, a excepción de los talleres que las asistencias son balanceadas, 50\% varones y la otra parte mujeres.

Las principales consecuencias están enfocadas a la destrucción de los recursos naturales y el medio ambiente de los pueblos indígenas, y a perder el territorio, la vigencia de una crisis según los niveles de intensidad del conflicto.

\section{Recomendaciones}

Las comunidades indígenas deben permanecer organizadas en sus estructuras tradicionales y promover movimientos sociales colectivos, sostenidos para la gestión de demandas y reclamos ante los gobiernos nacional, regional y municipal. 
La unidad de los pueblos indígenas es fundamental para garantizar esta reclamación histórica sobre la propiedad territorial ancestral, además se debe buscar la unidad con otras organizaciones de la sociedad civil para que conjuntamente se ejerza el respeto a los derechos de los indígenas.

Corresponde al Consejo Regional realizar el cabildeo e incidencia necesaria para limar asperezas entre el gobierno central, regional, municipal, territoriales y comunales, que permita agilizar la aplicación e implementación de la Ley No. 445.

En las comunidades indígenas debe promoverse la participación de las mujeres y la juventud en temas relacionados a la tenencia de la tierra.

En las estructuras de toma de decisiones de la CONADETI, hay una mujer, por lo que no podemos decir que la participación de la mujer en las estructuras de toma de decisiones en los distintos niveles es poco significante.

\section{Lista de referenciada}

Gabriel, Leo (1993). El universo de la tierra. Las culturas campesinas en el pacífico y centro de Nicaragua. Universidad Nacional Autónoma de Nicaragua. Edición Leo Gabriel.

Hale, Charles R. (1989). Diagnóstico general sobre la tenencia de la tierra en las comunidades indígenas de la Costa Atlántica. Consultoría No 084 - 96 Austin, Texas, Bluefields y Puerto Cabezas, Nicaragua, 1998 p. 78, 79, 80.

Hardin (1968). La tragedia de los Comunes. (s/n). WWW.eumed.net/cursecon/textos/ Hardin-tragedia.htm(consultado en 2005).

Kranz, Johnnes (2005). Introducción al estudio de conflictos interculturales. MAS - Bilwi Puerto Cabezas, RAAN pp. 3, 4.

Marroquín Mena, Edgar (1987). Evaluación sobre la reforma agraria en el Salvador (1980 - 1987). Cuaderno Universitario No. 15 Ciudad Universitaria, San Salvador, El Salvador p. 15-19.

Mclean Cornelio, Esther Melba (S /f). El caso Awas Tingni v. Nicaragua: Hacia el reconocimiento de los derechos de la propiedad comunal indígena en la Costa Atlántica (Nicaragua).

N. de Fernández M. y Cta., S. A (1972). Historia universal. Ediciones Eneva. Caracas. Venezuela p. 199 
Paredes Javier, et al., (1999). Historia Universal Contemporánea. I de las Revoluciones Liberales a la primera Guerra Mundial. España 1era ed. p. 27, 65, 68, 69, 81, $100,101,117$.

Roldan Ortega, Roque (1996). Derechos sobre las tierras de las comunidades indígenas de la Costa Atlántica. Informe final. Del trabajo de consultoría realizado en cumplimiento del contrato de trabajo OPA o62 - 96 INRA - Managua p. 31, 79, 80.

Stavenhagen, Rodolfo (2000). Los conflictos étnicos y sus repercusiones en la sociedad internacional. Htt. /www. Google. Com.ni www. UNESCO. Org /isssj /rics 157 Stavenhagen spa. htm/ - 67k p. 35, 36, 37, 40, 41.

Tarrow, Sidrey (1997). El poder en movimiento. Los movimientos sociales la acción colectiva y la política. Alianza Editorial. S. A. Madrid p. 77.

Vélez M. Laura, et al., (2000). Manual de entrenamiento básico en mediación. Instituto de Estudio y Promoción de la Autonomía (IEPA). Managua. Centro de mediación de conflictos México. Impreso en México. Av. Hidalgo 23753 -B p. 125, 126, 127.

Williamson, D. (1996). Investigación sobre Reforma Agraria en La Costa Atlántica. Central American and Caribean Research Council (CACRC). p. 80. 